\title{
SELF-INJECTIVE VON NEUMANN REGULAR SUBRINGS AND A THEOREM OF PERE MENAL
}

\author{
CARL FAITH ${ }^{1}$ \\ In memoria nobilissimi omnium-Pere
}

\begin{abstract}
This paper owes its origins to Pere Menal and his work on Von Neumann Regular (=VNR) rings; especially his work listed in the bibliography on when the tensor product $K=A \otimes_{K} B$ of two algebras over a field $k$ are right self-injective $(=S I)$ or $V N R$. Pere showed that then $A$ and $B$ both enjoy the same property, $S I$ or $V N R$, and furthermore that either $A$ and $B$ are algebraic algebras over $k$ (see [M]). This is connected with a lemma in the proof of the Hilbert Nullstellensatz, namely, a finite ring extension $K=k\left[a_{1}, \ldots, a_{n}\right]$ is a field only if $a_{1}, \ldots, a_{n}$ are algebraic over $k$.
\end{abstract}

In this paper, we follow Pere's lead in just the one property, namely $S I$, applied to a $V N R$ subring $A$ of a right $S I$ ring $K$. Pere proved in essence that all that is required is for $K$ to be split-fat over $A$ ( $=K$ is a flat left module and $A$ is a direct summand of $K$ as a right. $A$ module). By a theorem of Bourbaki-Lambek $[\mathbf{L}]$, the inclusion functor $\bmod -K \hookrightarrow \bmod -A$ preserves injectives iff ${ }_{A} K$ is fiat. Then (when $K_{K}$ is injcctive), $A_{A}$ is injective iff $A_{A}$ splits in $K_{A}$, equivalently, $A_{A}$ has no proper essential extensions in $K_{A}$ (cf. Proposition 1.1).

Pere's theorem on tensor products over an arbitrary commutative ring $k$ similarly can be generalized:

\footnotetext{
A part of this paper was written in Spring 1986 during my visit at Centre de Recerca Matemàtica (CRM) of the Institut d'Estudis Catalans in Universitat Autònoma de Barcelona (UAB). I owe much to Pere Menal for inviting me to visit UAB and CRM, and for our subsequent collaborations. (See my memento of Pere Menal i Brufal at the close of this article).
} 
Menal's general theorem. (1.7). If a right SI ring $K=A \otimes_{k} B$ is a tensor of faithful k-algebras $A$ and $B$ and is split-fiat over a commutative ring $k$, then $A$ and $B$ (also $k$ ) are right $S I$.

The proof requires a further bit of homological algebra $(1.3,1.4,1.5$ and 1.6). Moreover, Theorem 1.3 shows that only $B$ need be split flat over $k$ for the conclusion that $A$ is right $S I$.

The corollary that follows shows in one part of Pere's theorem [M] that the field $k$ can be replaced by any commutative self-injective $V N R$ $k$.

Corollary. (1.8). If $K=A \otimes_{k} B$ is right $S I$ faithful algebro over a $S I V N R$ ring $k$, then $A$ and $B$ are right $S I$.

The effect of the hypothesis that $K$ is faithful over $k$ is that $k \hookrightarrow K$, hence the $S I$ requirement for $k$ is equivalent to: $k$ has no proper essential extensions in $K$; and in this case $K$ is split over $k$. (And $K$ is flat because $k$ is $V N R$ ).

We next turn our attention to a maximal $V N R$ subring $A$, abbreviated $\max V N R$ in $K$. Then if $K$ is a right nonsingular module over $A$, by a theorem of $\mathrm{R}$. E. Johnson, $Q=Q_{\max }^{r}(R)$ embeds uniquely in $K$ (see, e.g., Lemma 3.1), hence cither $K=Q$, or else $A=Q$ is right $S I$. Expressed otherwise, when $K$ is nonsingular cither $A$ is right $S I$, or else $K$ is $V N R$ and $=Q$. Thus, $A$ is then right $S I$ assuming that $A$ is inessential or that $K$ is not $V N R$ (Corollaries $3.3 \mathrm{~A}-\mathrm{C}$ ).

Furthermore if $K$ is a $V N R$, even without assuming that $K$ is right nonsingular over $A$, we have:

Theorem. If $A$ is a $\max V N R$ in a $V N R$ right $S I$ ring $K \neq Q_{\max }^{r}(R)$, then $A$ is right $S I$ provided that $K$ is left strongly bounded $(=S B)$ in that sense that every left ideal $\neq 0$ contains an ideal $\neq 0$.

This theorcm follows from the preceding result for $K$ singuiar over $A$, without assuming $S B$; and otherwise from Theorem 5.5. We also have:

Theorem 5.6. If an Abelian $V N R$ ring $K$ is right $S I$, and right singular over a max VNR subring $A$, then $A$ is $S I$. Moreover,

$$
K \approx K_{1} \times A
$$

where $K_{1}$ is a skew field.

The next theorem follows from Utumi's theorem [U3], [U4] that characterizes a continuous $V N R$ ring $A$ by the property that $A$ contains all 
idempotents of $Q=Q_{\max }^{r}(A)$. (K Abelian in the theorem means that all idempotents are central).

Theorem 3.3E. A max VNR subring $A$ of a right self-injective Abelian (e.g., commutative) ring is continuous.

What happens when a $\max V N R$ subring $A$ does not contain all central idempotents of $K$ ? This is decided by Corollary 2.8: $K$ must be $V N R$ and either $K=Q$ or $A$ is right self-injective.

\section{Introduction}

\section{Wedderburn splitting and semisimple subrings.}

A number of conditions on a ring $K$ imply that a $V N R$ subring $A$ is right $S I$, without assuming that $K$ is right $S I$, or that $A$ is maximal, and we cite several below.

(I.1a) $K$ contains no infinite sets of orthogonal indempotents.

(I.1b) $K$ has the ascending condition on right (or left) annihilators, denoted acc $\perp$ (or $\perp a c c$ ),

(I.1c) $K$ is subring of a right (or left) Noetherian ring.

$$
\text { Note }(c) \Rightarrow(b) \Rightarrow \text { (a) for any subring } A \text {. }
$$

Furthermore, a $V N R$ ring $A$ with no infinite sets of orthogonal idempotents is semisimple Artin, and then every right or left $A$-module is injective.

(I.2) The center, cen $K$, of a $V N R$ is $V N R$, and, moreover, right $S I$ when $K$ is $V N R$ right $S I$ by a theorem of Armendariz-Steinberg [A-S].

(I.3) If $K$ is right $S I$, then by a theorem of Utumi [U], $\bar{K}=K / \mathrm{rad} K$ is right $S I$ and $V N R$. Thus any $V N R$ subring $A$ such that $K=A+(\operatorname{rad} K)$ is necessarily right self-injective inasmuch as $A \cap(\operatorname{rad} K=0$, hence $A \approx \bar{K}$. When this occurs, we say that $K$ has WEDDERBURN SPLITTING. For any (not necessarily $S I$ ) finite dimensional algebra $K$ over a field, this always occurs when $K$ is a separable algebra $([\mathrm{J}])$, and then $\vec{A}$ is semisimple by (1.1) above.

\section{Two cases of $\max V N R ' s$.}

The study of a $\max V N R$ subring $A$ in a ring $K$ devolves into two cases:

(II.1) A contains all central idempotents. This must occur if $K$ is not $V N R$,

(II.2) $K=A[e]=A+A e$ for a central idempotent $e$. 
In this case $K$ must be a VNR, and, moreover, a ring epic of $A^{2}$. (Theorem 4.5 and Corollary 4.3 ).

\section{Case 1: $A$ contains all central idempotents.}

(III.0) Theorem. Let $A$ be a subring of a ring $K$ that contains all central idempotents (e.g., suppose $A \supseteq \operatorname{Cen} K$ ).

(III.1) If $K$ is a ring product

$$
K=\prod_{i \in I} K_{i}
$$

then (III.0) implies:

$$
A=\prod_{\imath \in I} A_{\imath}
$$

where $A_{i}=A \cap K_{i} \forall i \in I$.

(III.2) Furthermore, $A$ is then $\max V N R$ in $K$ iff $A_{i}$ is $\max V N R$ in $K_{i} \forall i \in I$.

When $K$ is $V N R$, then $A$ is said to be a genuine $\max V N R$ in $K$ if $A$ is maximal in the set of all $V N R$ subrings $\neq K$.

(IrI.3) Corollary. If $A$ is a $V N R$ subring containing all central idempotents of a VNR ring $K$, then (III.1) holds, and then, $A$ is a genuine max $V N R$ of $K$ iff theTe exists $i_{0} \in I$ such that $A_{i_{0}}$ is a genuine $\max V N R$ of $K_{i_{0}}$ and $A_{2}=K_{i} \forall i \in I-\left\{i_{0}\right\}$. Moreover:

$$
K=Q_{\max }^{r}(A) \text { iff } K_{u_{0}}=Q_{\max }^{r}\left(A_{i_{0}}\right) .
$$

The proofs are trivial and are omitted.

To see how a $V N R$ right $S I$ ring $K$ decomposes into a ring product, see [G, p. 110]. If the right $S I$ ring $K$ is a $P I$-ring, then by a theorem of Armendariz and Steinberg [A-S], $K=\prod_{i \in I} K_{i}$, where each $K_{i}$ is a full matrix ring over an Abelian $V N R$ ring. (Cf. Theorem 5.3 in Section 5 below). Furthermore, in this case, $K$ is an Azumaya algebra over its center by theorems of $[\mathbf{A}-\mathbf{S}]$ and $[\mathbf{S}]$, see Theorem 2.4c.

Since $A+B$ is $V N R$ for any $V N R$ subring and $V N R$ ideal $B$ of $K$, then any $\max V N R A$ in a non $V N R$ ring $K$ must contain the maximal regular $(=V N R)$ ideal $M(K)$. Moreover, the implication (III.1) above then establishes: 
(III.4) Theorem. If $K=M(K) \times K_{2}$, where $M(K)$ is the maximal regular ideal, and $K_{2} \neq 0$, then any $\max V N R$ A splits:

$$
A=M(R) \times A_{2}
$$

where $A_{2}$ is a $\max V N R$ in $K_{2}$ and $M\left(K_{2}\right) \neq 0$.

Thus, by [F4], in the next corollary, we reduce the question of when $A$ is $S I$ to when $A_{2}$ is $S I$ :

(III.5) Corollary. If $K$ is a right and left SI ring that is not a $V N R$, then $K$ and any max VNR $A$ decompose as stated in (3.4). In this case, $M(K)$ and $K_{2}$ are 2-sided $S I$, and, moreover, $A$ is $S I$ iff $A_{2}$ is $S I$.

Actually, $M(K)$ splits off for any 2-sided continuous ring $K$ (see [F 4]). For a $V N R$ right $S I$ ring $K$, we apply a fundamental theorem of Utumi [U3] to decompose $K=K_{1} \times K_{2}$, such that $K_{1}$ is Abelian (= strongly regular), $K_{2}$ has no nonzero Abelian ideals, and $K_{2}$ is generated by idempotents.

This is a corollary to Theorem 2 of [U3], and is cited in [U4, Theorem 3.2].

(III.6) Theorem. If $K$ is a VNR right SI ring, not a centralizing extension of a genuine max $V N R A$, then the stated Utumi decomposition induces a decomposition $A=A_{1} \times A_{2}$, where $A_{i}=A \cap K_{i}$ is a genuine max $V N R$ for exactly one $i \in\{1,2\}$, and $A_{j}=K_{j}$ for $j \neq i, j=1$, or 2 .

\section{Case 2: Does not contain all central idempotents.}

In this case, $K=A[e]=A=e A$ for a central idempotent $e$, hence $K$ must be $V N R$, and, as it happens, a ring epic of $A^{2}$ (Theorem 4.5 and Corollary 4.3). Furthermore, once again, we find that $A$ is right $S I$ when $K \neq Q$ :

(IV.1) Theorem. (2.7) If $K$ is a right self-injective VNR, and if $K$ is a centralizing extension of a genuine max $V N R A$, then either $A$ is right $S I$;

or

$$
K=Q_{\max }^{r}(A) .
$$


(IV.2) Corollary. Under the same assumptions, then $A$ is right $S I$ iff $A$ is inessential in $K$ os a right $A$-module.

We next show that $A$ need not be right $S I$ in some cascs.

(IV.3) Example. (Cf. (4.10)) If $R$ is any subring of a right selfinjective prime ring $K$, and if $R$ contains a nonzero left ideal $H$ of $K$, then $K=Q=Q_{\max }^{r}(R)$.

Proof: If $0 \neq a, b \in K$, then $a L \neq 0$ by primeness of $K$. Then, there exists $x \in L \subseteq R$ with $0 \neq a x \in L \subseteq R$ and $b x \subseteq R$. So $K=Q$. (See, e.g., [F, Chapter 8]). If follows that if $A$ is any $V N R$ subring of $K$ containing $R$, then $A$ is right $S I$ iff $A=K$.

To give a specific example let $K=$ End $V_{D}$ for a right vector space $V$ over a field $D$, and let $H$ be the right socle of $K$ (= the sum of all minimal right idcals). Since $H$ is an essential right idcal, and $V N R$; then $R=D+H$ is $V N R$ and essential in $K$ as a right $R$-module, then $Q_{\max }^{r}(R)=K$ for any $V N R$ subring $A \supseteq R$. Thus, no genuine max $V N R$ containing $R$ can be right $S I$.

In Theorem 5.7 we record the following curiosity: if a genuine max $V N R$ subring $A$ does not contain a maximal ideal of a right self-injective $V N R$ ring $K$, then $K$ is a subdircet product of simple homomorphic images of $A$ (cf. Corollary 4.3).

\section{Acknowledgements.}

Thanks to Ken Goodearl for pointing out that Corollary 7 of [F3] requires the nonsingular hypothesis of the preceding Theorem 6 . (This was left out inadvertently).

Grateful thanks to Manuel Castellet, the Director of CRM, for inviting me to CRM and for authorizing the Algebra semesters of Spring 1982 and Fall 1989.

'To the amazing and beautiful Elena Carles, I am immensely indebted for her many efforts on behalf of my wife, Molly Sullivan and myself, and for helping to make our tenurcs in Catalunya so pleasant, and (for mc) mathematically productive.

Finally, one can not say enough in praise of Pere Menal's students who contributed so much to the exciting mathematical atmosphere at UAB and CRM, not to say the jolly fun they generate at any occasion! (See Section 7).

Added in Proof. A theorem of Pere's, given by Pere's student, Claudi Busqué in [B], shows that any $S I V N R$ ring $R$ is a product of algebras over fields. This may be useful in studying the sequel. 


\section{Self-injective split-flat extensions, algebras and tensor products}

If $A$ is a subring of $K$ then is a split (resp. flat) extension provided that $A$ is isomorphic to a direct summand of $K$ as a right $A$-module (resp., $K$ is a flat left $A$-module). Thus, by a theorem of Azumaya, $K$ is a split extension iff $K$ generates mod- $A$. (See [F5, p. 145, Cor. $3.27(\mathrm{a}) \mid)$. Furthermore, $K$ is said to be split-flat over $A$, if $K$ is both a split and flat extension of $A$. If $K$ is an algebra over a commutative ring $k$, then $K$ is a split (flat) algebra if $K$ is a split (flat) extension of $k$. This implics, in either case, that $K$ is faithful over $k$, equivalently that the canonical map $k \rightarrow K$ is an embedding.

1.1. Proposition. If $K$ is right self-injective, and if $K$ is a flat left $A$-module for a subring $A$, then $K$ is an injective right $A$-module. Moreover, in this case $A$ is right self-injective iff $K$ is right split over $A$, equivalently, $A$ is a direct summand of $K$ in mod- $A$.

Proof: Sec [F3].

1.2A. Corollary. Let $K$ be right self-injective, and $G$ be a finite group of automorphisms of unit order. Then, if $A=K^{C}$ is $V N R$, it is right self injective.

Proof: $K$ is flat over $A$ since $A$ is $V N R$, and a splits in $K$ (both sides) by Maschke's theorem (e.g., see [F5, p. 475, Theorem 13.21]).

The next result generajizes a theorem of $[\mathrm{P}]$. (See Open Questions, Section 6).

1.2B. Corollary. If a VNR $K$ is right $S I$, and $G$ is a group of automorphism of unit order, then the fix ring $A=K^{G}$ is right $S I$.

1.3. Theorem. If $A$ and $B$ are algebras over $k$, and if $K=A \otimes_{K} B$ is right self-injective, and if $B$ is a splat-flat algebra over $k$, then $A$ is right self-injective.

Proof: Since $k$ splits in $A$, then $A \approx A \otimes_{k} k$ splits in $K$. Spccifically: if $B \approx k \oplus X$ in mod-k, then for $Y=A \otimes_{k} X$

$$
K \approx A \otimes_{k} B \approx\left(A \otimes_{k} k\right) \oplus\left(A \otimes_{k} X\right) \approx A \oplus Y
$$

in mod- $A$. Moreover, $K$ is left flat over $A$, since if $0 \rightarrow U \rightarrow V$ is exact in $\bmod -A$, then

$$
U \otimes_{A} K \approx U \otimes_{A}\left(A \otimes_{k} B\right) \approx\left(U \otimes_{A} A\right) \otimes_{k} B \approx U \otimes_{k} B .
$$


Since $B$ is flat over $k$, then

$$
0 \rightarrow U \otimes_{k} B \rightarrow V \otimes_{k} B
$$

is exact, hence

$$
0 \rightarrow U \otimes_{A} K \rightarrow V \otimes_{A} K
$$

is exact.

Since $K$ is therefore left flat and a right generator over $A$, then by Proposition 1.1, $A$ is right self-injective.

1.4. Lemma. If $K=A \otimes_{k} B$ is a flat $k$-algebra, and if $\otimes_{k} B$ is a faithful functor, then $A$ is a flat $k$-module. A sufficient condition for faithfulness of $\otimes_{k} B$ is for $B$ to be a $k$-split algebra.

Proof: Let $0 \rightarrow X \rightarrow Y$ be exact in mod- $R$, and let

$$
0 \longrightarrow U \longrightarrow X \otimes_{k} A \stackrel{\text { can }}{\longrightarrow} Y \otimes_{k} A
$$

be exact. Then

$$
0 \longrightarrow U \otimes_{k} B \longrightarrow X \otimes_{k} K \longrightarrow Y \otimes_{k} K
$$

is exact, but fatness of $K$ then implies that $U \otimes_{k} B=0$, whence $U=0$ by faithfulness of ${ }_{k} B$, so $0 \rightarrow X \otimes_{k} A \rightarrow Y \otimes_{k} A$ is exact. This proves that $A$ is fiat over $k$.

Next suppose that $B$ is split over $R, k \approx R \oplus X$ in mod- $R$. Then obviously $\otimes_{k} B$ is faithful, since it has a faithful subfunctor: $\otimes_{k} \approx 1_{\text {mod- } R}$.

1.5. Theorem. A tensor product $A \otimes_{k} B$ of modules over a commutative ring $k$ generates mod-k iff both $A$ and $B$ generate mod- $k$.

Proof: A module $A$ generates mod- $k$ iff the trace ideal $T_{k}(A)=k$, where for a $k$-module $A, T_{k}(A)$ is the image of the canonical map $A^{*} \otimes_{k}$ $A \rightarrow k$, where $A^{*}$ is the $k$-dual module of $A$. Now, tensoring over $k$, we have

$$
\begin{aligned}
K^{*} \otimes K & =A^{*} \otimes B^{*} \otimes A \otimes B \\
& =\left(A^{*} \otimes A\right) \otimes\left(B^{*} \otimes B\right)
\end{aligned}
$$

hence the trace ideal

$$
T(K)=T(A) T(B) .
$$

For, if $\varphi_{1}: A^{*} \otimes A \rightarrow k$ and $\varphi_{2}: B^{*} \otimes B \rightarrow k$ are canonical, then the canonical map $K^{*} \otimes K \rightarrow k$ is $\varphi=\varphi_{1} \otimes \varphi_{2}$ and so

$$
T(K)=\operatorname{im} \varphi=\varphi_{1} \otimes \operatorname{im} \varphi_{2}=T(A) T(B)
$$

it follows that $T(K)=k$ iff $T(A)=T(B)=k$, proving the theorem. 
1.6. Corollary. If $K=A \otimes_{k} B$ is a split algebra over $k$, then so are $A$ and $B$. Moreover, if $K$ is a split-fiat algebra, then so are $A$ and $B$.

Proof: $A$ and $B$ are split algebras by Theorem 1.5 and Azumaya's Theorem cited at the beginning of this section and then $A$ and $B$ are Rat algebras by Lemma 1.4 .

1.7. Menal's General Tensor Theorem. If $K$ is a right selfinjective split-flat algebra over a commutative ring $k$, and if $K=A \otimes_{k} B$, for subolgebras $A$ and $B$, then $A$ and $B$ are right self-injective split-flat algebras.

Proof: Corollary 1.6 and Theorem 1.3.

1.8. Corollary. If $K=A \otimes_{k} B$ is a right $S I$ faithful algebra over a $S I$ commutative $V N R k$, then $A$ and $B$ are right $S I$.

Proof: $K$ is split-flat over $k$, so Menal's General Theorem applies.

\section{Azumaya subalgebras and centralizing extensions}

In this section we demonstrate that an Azumaya algebra $A$ over $k$ is (right and left) self-injective if $A$ embeds in a right self-injective split-flat $k$-algebra. This follows from the more general theorem (Theorem 1.7) stating that if $K=A \otimes_{k} B$ is right self-injective "split-fat" algebra over $k$ then $A$ and $B$ are right, self-injective, and hence, then so is $k$.

We also note that any self-injective ring $V N R$ ring $K$ with polynomial identity $(=P I)$ is Azumayan over its center (Theorem 2.4C). This follows easily from $[\mathrm{A}-\mathrm{S}]$ and $[\mathrm{S}]$.

For use below, if $A$ is an algebra over a commutative ring $k$, the enveloping algebra $A^{e}$ is defined to be the tensor product $A \otimes_{k} A^{0}$ of $A$ and the opposite algebra $A^{0}$. Then the operation defined by the rule $X\left(a \otimes b^{0}\right)=b x a$ for any $a, b, x \in A$ defines $A$ as a canonical (right) $A^{e}$-module. There is a canonical defined by $h: A^{e} \rightarrow \operatorname{End}_{k} A$

$$
x h\left(a \otimes b^{0}\right)=b x a
$$

and the image of $h$ is the subring $A_{d}\left[A_{s}\right]$ of $\mathrm{End}_{k} A$ generated by the subring $A_{d}$ consisting of all right, and the subring $A_{s}$ consisting of all left, multiplications of the $k$-module $A$ by elements of $A$. 
2.0. Azumaya algebra definition and Theorem. An algebra $A$ over a commutative ring $k$ is called an Azumaya algebra if $A$ satisfies the equivalent conditions.

(Az 1) $A$ is a projective module over the enveloping algebra $A^{e}=$ $A \otimes_{k} A^{0}$.

(Az 2) $A$ is a finitely generated projective module over $k$, and $A^{e}=$ End $_{k} A$ canonically.

(Az 3) $A^{e}$ is Morita equivalent to $k$.

(Az 4) $A$ is finitely generated module over $k$, and for all maximal ideals $m$ of $k$, the factor algebra $A / m A$ is a central simple $k / m$-algebra.

(Az 5) $A$ is finitely generated projective and central over $k$ and every ideal $I$ of $A$ is of the form $I=I_{0} A$, where $I_{0}=I \cap k$.

(Az 6) A generates $\bmod -A^{e}$ and $k=\operatorname{End}_{A^{c}} A$.

When this is so, then Cen $A=k$.

Proof: Most of this is due to Azumaya ( $\mathrm{Az} 1$ ) over local $k$, and the carry-over to general $k$ is in $[\mathbf{A}-\mathrm{O}]$. Also see $[\mathbf{B}]$.

The equivalence of $(A z 5)$ with $(A z 1)$ is a theorem of Rao [R]. Moreover, the Morita equivalence (Az 3) implies (Az 6). Conversely (Az 6) $\Rightarrow(\mathrm{Az} 2),(\mathrm{Az}$ 6) $\Rightarrow(\mathrm{Az}$ 2) a theorem of Morita ([F5, p. 190, Theorem 4.1]) and Azumaya (in [Az]).

2.1. Proposition. If $A$ is an Azumaya algebra over a commutative. ring $k$, then the f.a.e.

(2.1.A) $A$ is right self-injective.

(2.1.B) $A$ is left self-injective.

(2.1.C) $k$ is self-injective.

(2.1.D) $A^{e}$ is right self-injective.

(2.1.E) $A^{e}$ is self-injective.

Proof: $k$ is Morita equivalent to $A^{e}$, hence $A^{e}$ is right self-injective iff $k$ is self-injective, and this implies $A^{e}$ is then left self-injective. Moreover, since Azumaya algebras are flat generators over $k$ (in fact, finitely generated faithful projective modules over $k$ (in fact, finitely generated faithful projective modules over $k$ ), then $A^{e} \approx A \otimes_{k} A^{0}$ implies via Theorem 1.3 (or Theorem 1.7) that $A$ and $A^{0}$ are self-injective (both sides).

2.2. Azumaya Theorem. If $A$ is an Azumaya algebra over a commutative ring $k$, and if $A$ is a subalgebra of an algebra $K$ over $k$, then $K \approx A \otimes_{k} A^{\prime}$, where $A^{\prime}$ is the centralizer of $A$ in $K$.

Proof: [B, p. III-28, Corollary 4.3]. 
2.3. Corollary. If $K$ is right self-injective, and if a subring $A$ is an Azumaya algebra over a subring $k$ of $C=\operatorname{Cen} K$, then (a) $A$ is right self-injective, (b) $k$ is self-injective, and (c) $A$ is left self-injective.

Proof: $K=A \otimes_{k} A^{\prime}$ by the theorem, and then $A$ and $A^{\prime}$ are right self-injective by Theorem 1.7. Then $k$, also $A$ on the left, is self-injective by Proposition 2.1 .

We need a now classical result.

2.4A. Lemma (Azumaya). If $K$ is an Azumaya algebra over $k$, then for any proper ideal $I$ of $K$, the subalgebra $K / I$ is central over $R /(I \cap R)$.

Proof: Suppose that $x \in K$ and that $x y-y x \in I$ for all $y \in K$. Let $m$ be a maximal ideal of $k$ so that $m K \supseteq I$. Then $\bar{K}=K / m K$ is central simple over $\bar{k}=k / m k$, consequently $\bar{x} \in \bar{k}$ proving that $x \in k$. This shows that $k / k \cap I$ is the center of $K / I$.

2.4B. Proposition. If $K$ is an Azumaya algebra over $k$, and if $A$ is a (max) VNR subring, then $A \cap k$ is $V N R$.

Proof: Write $A_{0}=A \cap R$, and let $a \in A_{0}$. Then, by regularity of $A$, there exists $x \in A$ such that axa $=a$. Since $a \in k$, then $x a^{2}=a$, if $y \in K$, then

$$
y x a^{2}=y a=a y=x a^{2} y=x y a^{2}
$$

so $y x-x y$ annihilates $a^{2} K=a K$. This implies that in $K / a K$ that $x$ maps onto an element of the center of $K / a K$, so the lemma implies that $x \in k$. Thus, $x \in A_{0}$ proving that $A_{0}$ is $V N R$.

A ring $K$ is biregular provided that for all $a \in K$ every principal ideal $K a K$ is generated by a central idempotent. Evidently, every simple ring is biregular, i.e., a biregular ring need not be regular.

We note the following characterization of Azumaya algebras over $V N R$ 's.

Szeto's Theorem. ([S]) A ring $K$ is an Azumaya algebra over a commutative $V N R$ subring $C$ iff $K$ is a biregular ring finitely generated as a module over its center $C$.

We also note: 
Renault's Theorem. ([Re2]). AVNR right SI ring $K$ is biregular iff every prime ideal is maximal.

Cf. [Re2, Proposition 3.7], which states that $Q_{\max }^{r}(R)$ is biregular when $R$ is reduced.

2.4C. Theorem. A VNR right SI ring $K$ with $P I$ is an Azumaya algebra over its center $k$.

Proof: The center $k$ of $K$ is a VNR ring, and by [A-S, Theorem 3.7 and Coroliary 3.2]) $K$ is a biregular ring that is a finitely generated projective module over its center $k$. It follows from Szeto's Theorem that $K$ is an Azumaya algebra over $k$.

2.4D. Notes. 1. Theorem $2.4 \mathrm{C}$ is implicit in $[\mathrm{A}-\mathrm{S}]$ where the ideal correspondence

$$
\phi \begin{cases}\text { Ideals } k & \longrightarrow \text { Ideals } K \\ I & \longrightarrow I K\end{cases}
$$

is proved a bijection with $\phi^{-1}(J)=J \cap k$ for an ideal $J$ of $K$. This is a consequence of the biregularity of $K$ see $[\mathbf{A}-\mathbf{S}]$, footnote added in proof. (Also see [A-S, Corollary 3.6], where the ideal correspondence is proved using the fact that $K$ is a direct sum of finitely many Azumaya algebras!).

2. Using 1 , Theorem $2.4 \mathrm{C}$ also follows from (Az 5$)$ of Theorem 2.0 .

2.4E. Corollary. Let $A$ be a VNR central subalgebra of a VNR right $S I$ algebra $K$ with $P I$ over its center $k$. Then $A$ is self-injective iff $A$ is an Azumaya algebra over $k$.

Proof: If $A$ is $\Lambda$ zumaya over $k$, then $A$ is $S I$ by Corollary 2.3. Conversely, if $A$ is $S I$, then $A$ is Azumaya over $k$ by Theorem $2.4 \mathrm{C}$.

2.4F. Remark. In [A-S], the PI's have coefficients in the centroid of $K$, but since $K$ is $S I$, the centroid is $k$. (At least one coefficient must be a unit).

\section{Centralizing extensions.}

An ring $K$ is a central extension of a subring $A$ provided that $K$ is the subring $A[C]$ generated by $A$ and $C=\operatorname{Cen} K$. If $K=A\left[A^{\prime}\right]$ is generated by $A$ and its centralizer $A^{\prime}=\operatorname{Cen}_{K} A$, then we say that $K$ is a centralizing extension of $A$. By Theorem $2.1, K$ is a centralizing extension of any subalgebra that is Azumaya over a subring $k$ of Cen $K$.

An idempotent $e \in K$ centralizes a subring $A$ if, $a$ belongs to the centralizer of $A$ in $K$. 
2.5. Lemma. If $A$ is a $V N R$ subring of a ring $R$, then so is $A[e]=$ $A+$ Ae for any idempotent $e$ of $K$ that centralizes $A$.

Proof: A ring $B$ is $V N R$ if there exists a $V N R$ ideal $I$ so that $B / I$ is $V N R$. (In this case every factor ring and every ideal is $V N R$ ). In our case, let $B=A+A e$, and then $I=A e$, is an ideal of $B$ and is a $V N R$ ring since it is a homomorphic image of $A$. Moreover, $B / I \approx A /(I \cap A)$ is also a homomorphic image of $A$, so $B$ is $V N R$.

If $N \subseteq M$ are $A$-modules, then $N \subset M$ denotes that $N$ is an essential submodule of $M$.

If $M$ is a right $A$-module, then the singular submodule $\operatorname{sing} M_{A}$ is defined by

$$
\operatorname{sing} M_{A}=\left\{m \in M \mid \operatorname{ann}_{A} m \underset{\text { ess }}{\subset} A\right\}
$$

where, as stated,

$$
\operatorname{ann}_{A} m=\{a \in A \mid m a=0\} .
$$

Now $S=\operatorname{sing} M_{A}$ is a fully invariant submodule of $M$, that is, $b s \in S$ for all $b \in$ End $M_{A}$ and all $s \in S$. This implies that $\operatorname{sing} A_{A}$ is an ideal. If $\operatorname{sing} M_{A}=0$, then $M$ is said to be nonsingular, otherwise singular.

2.6A. Lemma. If $K$ is a ring and $A$ is a subring, then the right singular $A$-submodule $S=\operatorname{sing} K_{A}$ is a $(K, A)$-submodule of $K$, hence a left ideal of $K$.

Proof: $S$ is a fully invariant $A$-submodule of $K$.

2.6. Proposition. If $K$ is a right self-injective ring and right nonsingular over a subring $A$, then $S=\operatorname{sing} K_{A}$ is an ideal of $K$ under any of the conditions:

(1) $K$ is a central extension of $A$.

(2) $K=A\left[A^{\prime}\right]$ is the subring generated by $A$ and the centralizer $A^{\prime}$ of $A$ in $K$.

(3) $A \subseteq C=\operatorname{Cen} K$.

Proof: Since $S$ is a $(K, A)$-submodule, then $S$ is an ideal iff $S K \subseteq S$. It suffices to prove (2), since $A^{\prime} \supseteq C$ so $K=A[C] \Rightarrow K=A\left[A^{\prime}\right]$. Also, $A \subseteq C \Rightarrow A^{\prime}=K$ so $K=A\left[A^{\prime}\right]$ in this case too.

(2) If $b \in A^{\prime}$ and $s \in S$, then

$$
s b\left(s^{\perp} \cap A\right)=s\left(s^{\perp} \cap A\right) b=0
$$

so $s b \in S$, that is, $S$ is a right $A\left[A^{\prime}\right]$-submodule of $K$. Then $K=A\left[A^{\prime}\right]$ implies that $S$ is an ideal. 
2.7. Theorem. If $K$ is a right self-injective $V N R$, and a centrolizing extension of a genuine max $V N R$ subring $A$, then either

(1) $A$ is right self-injective; or else

(2) $K=Q_{\text {rrax }}^{r}(A)$

Proof: If $K=A\left[A^{\prime}\right]$ is a centralizing extension of $A$, then $S$ is an ideal of $K$, hence $S$ is a $V N R$ ring-1. If $S \neq 0$, then $S+A$ is a $V N R$ subring properly $A$, so $K=S+A$ by maximality of $A$. Thus, $A$ splits in $K$ in $\bmod -A$ (also in $A$-mod), so $A$ is injective by Proposition 2.1. If $S=0$, then Corollary $2.3 A$ applies.

2.8. Corollary. If $K$ is right self-injective, and if $A$ is a genuine maximal $V N R$ not containing all central (or centralizing) idempotents in $K$, then $K$ is a $V N R$ ring, and either $A$ is right self-injective, or else $K=Q_{\max }^{r}(A)=A+A e$, for a central idempotent e.

Proof: Suppose that $e=e^{2}$ belong to the centralizer $A^{\prime}$ of $A$ in $K$ but non in $A$. Then, since $A+A e$ is a regular ring by Lemma 2.5 , $K=A+e A=A[e]$ is a central extension of $A$, so the theorem applies.

\section{Self-injective rings nonsingular over regular subrings}

This section is mainly devoted to rings described by the section title, but in some instances other conditions are considered, namely $K$ flat over a nonsingular subring $A$, or $K$ Galois over regular subrings, without assuming $K$ nonsingular over $A$.

We also consider dense flat maximal subrings of self-injective rings.

If $M \supset N$ are modules over $A$, and if $x \in M$, then

$$
(x: N)=\{a \in A \mid x a \in N\}
$$

is the conductor of $x$ in $N$. If $N$ is an essential submodule of $M$, then $(x: N)$ is an essential right ideal of $A$. The annihilator of $x$ in $A$ is $(x: 0)$, also denoted by $\mathrm{ann}_{A} x$.

A subring $A$ of $K$ is left (right) essential if $A \underset{e s s}{\subset} K$ as a left (right) $A$-module. Otherwise $A$ is lefi (right) inessential ins $K$.

3.1. Lemma. If a right self-injective ring $K$ is right nonsingular over a VNR subring $A$, then $Q=Q_{\max }^{r}(A)$ embeds uniquely as a subring of $K$ 
containing $A$. A sufficient condition is that $A$ is a right or left essential $V N R$ subring of $K$.

Proof: This is essentially a theorem of R. E. Johnson (see, e.g. [F, Chapter 8]). We give a proof here for completeness and comprehensibility. By a theorem of Auslander-Harada, every (right and left) $A$ module is flat (see, e.g. [F5, p. 434, Theorem 11.4]). By Lemma 2.6A, $S=\operatorname{sing} K_{A}$ is a $(K, A)$-submodule of $K$. Since $S \cap A=0$ by nonsingularity of $A$, then $S=0$, when $A$ is left or right essential in $K$. In this case, $K$ is right non-singular over $A$.

Since $K$ is (right and left) flat over $A$, by Theorem 1.1 then $K$ is injective in mod- $A$, hence contains an injective hull $E(A)=E$ of $A$. We first prove that $E$ is the unique injective hull of $A$ contained in $K$ : If $F$ were another one, then there is an isomorphism $f: E \rightarrow F$ of $A$ modules. But if $x \in E$, the conductor of $x$ in $A$ is an essential right ideal $I$ (notation $I=(x: A)$ ) and

$$
x a=f(x a)=f(x) a \forall a \in I
$$

so $x-f(x)$ annihilates $I$, hence belongs to the singular submodule $\operatorname{sing}_{A} K$. Since $K$ is nonsingular, then sing ${ }_{A} K=0$, so $f(x)=x \forall x \in E$, hence $F=E$.

Now as in [F, Chapter 8, Theorems 1 and 2], $E$ has the structure of the maximal quotient ring $Q=Q_{\max }(A)$, so it remains to show that the ring product $x \cdot y$ in $Q$ coincides with that in $K$ for all $x, y \in E$. To do this if $I$ the conductor of $y$ in $A$, then

$$
(x \cdot y) a=x(y a)=x(y a)=(x y) a
$$

so $x \cdot y-x y$ annihilates an essential right ideal $I$, hence $x \cdot y-x y \in$ $\operatorname{sing} K_{A}=0$. This proves that $x \cdot y=x y \forall x, y \in E$ and therefore $Q$ embeds in $K$.

3.2. Proposition. If $A$ is a semisimple subring of a ring $K$, then $K$ is right and left nonsingular over $A$.

Proof: The only essential one-sided ideal of a semisimple ring $A$ is $A$. Since $1 \in A$, then both right and left $A$-singular $A$ submodules of $K$ are zero.

3.3A. Corollary. If $K$ is a right self-injective ring right nonsingular over a maximal VNR $A$, then either $K=Q_{\max }^{r}(A)$, in which case $K$ is $V N R$, or $A$ is right self-injective. 
Proof: $K$ is right nonsingular over $A$, so $Q=Q_{\max }^{r}(A)$ embeds in $K$ by the theorem. However, $Q$ is a regular right self-injective ring, so either $Q=K$, or else $Q=A$ is right self-injective.

3.3B. Corollary. If $A$ is a right essential $V N R$ subring of a right self-injective ring $K$, then $K=Q_{\max }^{r}(A)$, hence $K$ is $V N R$.

Proof: $A$ is right essential, hence $K$ is a nonsingular right $A$-module, so Lemma 3.1 applies, that is, $K \supseteq Q_{\max }^{r}(A)$, hence $K=Q_{\max }^{r}(A)$.

3.3C. Corollary. If $K$ is a right self-injective ring right nonsingular over a max $V N R A$, and if either $K$ is not $V N R$ or $A$ is right inessential, then $A$ is right self-injective.

Proof: In either case, $K \neq Q$, hence $A=Q$ by Corollary 3.3A.

3.3D. Corollary, Let $K$ be right SI. If every nonzero left ideal of $K$ contains a nonzero central idempotent then $K$ is right nonsingular over any right nonsingular subring $A$ containing all central idempotents, hence the conclusions of Corollary $3.3 \mathrm{C}$ hold.

Proof: The right singular submodule $S=\operatorname{sing} K_{A}$ is a left ideal of $K$ satisfies $S \cap A=0$ since $A$ is right nonsingular. But $S \neq 0$ implies the existence of a central idempotent $e \neq 0$ in $S$ which violates $S \cap A=0$.

If every idempotent of top $K=K / J$ lifts to an idempotent of $K$, we say that top idempotents lift. A ring $K$ is Abelian if all idempotents are central.

3.3E. Theorem. If $K$ is an Abelian self-injective ring, then a max $V N R A$ is continuous.

Proof: We may assume $K$ is not $V N R$. By Lemma $2.5, A$ contains all idempotents. By a theorem of Utumi [U1], all top idempotents of a selfinjective ring lift, so $\bar{A}$ contains all idempotents of $\bar{K}=K / J$. By Lemma $3.3 \mathrm{D}, \ddot{K}$ is nonsingular over $\bar{A} \approx A$, hence contains $Q_{\max }(\bar{A}) \approx Q_{\max }(A)$, and therefore $A$ is continuous by Utumi's Theorem ([U3], [U4]).

3.3F. Corollary. If $K$ is a right self-injective ring nonsingular over a maximal commutative $V N R A$, then either $K=Q_{\max }^{r}(A)$, or else $A$ is self-injective.

Proof: By Theorem 3.1, $Q=Q_{\max }(A)$ embeds in $K$. Since $Q$ is commutative $V N R$ and self-injective, then by maximality of $A$, wither $K=Q$ or $A=Q$. Since $Q$ is $S I$, the latter implies that $A$ is. 
3.3G. Corollary. If $K$ is a right $S I$ ring that is right nonsingular over a max commutative $V N R A$, and if either (1) $K$ is noncommutative or (2) $K$ is not $V N R$, or (3) $A$ is inessential, then $A$ is $S I$.

Proof: Same as Corollary 3.3C.

3.3H. If a max commutative $V N R A$ is right or left essential in a right $S I$ ring $K$ then $K=Q_{\text {rrax }}(A)$ is commutative.

Proof: $K$ is right nonsingular over $A$ by Theorcm 3.3. Since $A$ is essential but $\neq K$, then $A$ camnot be injective, hence $A \neq Q_{\max }(A)=K$, by Corollary 3.3D.

\section{Central idempotents and direct products}

In this chapter we prove theorems on a maximal $V N R$ subring $A$ of a direct product $K=\Pi_{\alpha \in \Lambda} K_{\alpha}$.

The first result restates Theorem (III.0) of the Introduction.

4.1. Trivial Lemma. 1. If a subring A contains all central idempotents of a ring $K$, then any direct product representation $K=\Pi_{\alpha \in \Lambda} K_{\alpha}$ induces a direct product representation $A=\Pi_{\alpha \in \Lambda} A_{\alpha}$ where $A_{\alpha}=K_{\alpha} \cap A$. (If $e_{\alpha}$ is the unit element of $K_{\alpha^{\prime}}$ then $A_{\alpha}=e_{\alpha} A$ ).

2. If $K$ is $V N R$, then $A$ is genuine $\max V N R$ in $K$ iff there exists $\beta \in \Lambda$ such that $A_{\alpha}=K_{\alpha}$ for all $\alpha$ except $\alpha=\beta$ and $A_{\beta}$ is a genuine $\max V N R$ in $K_{\beta}$.

4.2. Theorem. Let $A$ be a genuine $\max V N R$ of $K$, and lel e be any central idempotent.

(1) If $e \notin A$, then $e A=e K$ and $(1-e) A=(1-e) K$, and

$$
K \approx e A \times(1-e) A \approx A /(A \cap(1-e) A) \times A / A \cap e A .
$$

(2) If $e \in A$, then either (2a) or (2b) holds:

(2a) $e K=e A$, whence

$$
K=e A \times(1-e) K
$$

(2b) $(1-e) K=(1-e) A$ whence

$$
K=e K \times(1-e) A .
$$

Moreover, any idempotent $e \in \mathrm{Cen}_{K} A$ is central. 
Proof: By Lemma 2.5, $B=A+e A$ is a regular ring, hence in case $e \notin A$ necessarily $B=K$ by maximality of $A$. The rest of (1) is evident.

Since $K \neq A$, then either $e K \neq e A$ or $(1-e) K \neq(1-e) A$, when $e \in C \cap A$. Hence in case $(1-e) K \neq(1-e) A$, then by Lemma 2.5 , $B=A+(1-e) K$ is a $V N R$ subring properly containing $A$, hence $B=K$, so (2a) holds, and (2b) holds in the contrary case.

Now let $e \in \operatorname{Cen}_{K} A$. One easily shows that $B_{1}=e K e+A$ is a $V N R$ subring containing $A$, and similarly for $B_{2}=(1-e) K(1-e)+A$. If $B_{1} \neq A$, then $K=e K e+A$, hence $(1-e) K=(1-e) A$ so $(1-e) K e=0$. By symmetry $e K(1-e)=0$, so $e$ is central. Similarly when $B_{2} \neq A$, then $1-e$, whence $e$, is central. Finally $B_{1}=B_{2}=A$ is impossible since $A \neq K$.

4.3. Corollary. Let $A$ be a genuine $\max V N R$ of a $V N R$ ring $K$, and let e be a central idempotent. If $e \notin A$, then $K$ is an epic image of $A^{2}$, hence is $V N R$; and if $e \in A$, then either $e A$ or $(1-e) A$ is a direct factor of $K$.

4.4. Theorem. If $A$ is a genuine maximal subring of a $V N R$ ring $K$. Let $K=K_{1} \times K_{2}$, and let $e_{i}$ denote the identity of $K_{i}, i=1,2$. If one $e_{i} \notin A$, say $e_{1} \notin A$, then $e_{i} A=K_{i}, i=1,2$, so

$$
K=e_{1} A \times e_{2} A \text {. }
$$

Moreover, if one hence both $e_{i} \in A, i=1,2$, then

$$
K=e_{1} A \times K_{2}
$$

or

$$
K=K_{1} \times e_{2} A .
$$

In (2a), $e_{1} A$ is a genuine $\max V N R$ of $K_{1^{\prime}}$, and in $(2 \mathrm{~b}), e_{2} A$ is a genuine $\max V N R$ of $K_{2}$.

Proof: Follows from Theorem 4.2 and the Trivial Lemma 4.1 .

4.5. Theorem. If $A$ is a maximal (commutative) VNR subring of a non- $V N R$ ring $K$, then $A$ contains all central idempotents, and moreover all idempotents of $K$ that centralize $A$.

Proof: By Lemma $2.5, B=A+A e$ is a $V N R$ subring of $K$, hence $B=A$ by maximality (also in case $A$ is a maximal commutative $V N R$ subring), so $e \in A$ for all idempotents $e \in A^{\prime}$. Since $A^{\prime} \supset C=\operatorname{cen} K$, then $A$ contains all central idempotents. 
4.6. Corollary. If $A$ is a maximal (commutative) VNR subring of a non- $V N R$ ring $K$, and if $C=$ Cen $K$ is generated by idempotents then $A \supseteq C$. Moreover, if $A^{\prime}$ is generated by idempotents, then $A \supseteq A^{\prime}$. In case $A$ is right $S I$, then so is $A^{\prime}$ in the latter case.

Proof: If $A^{\prime}$ is generated by idempotents, then $A \supseteq A^{\prime}$ by the theorem. If $A$ is right $S I$, then so is $A^{\prime}=\operatorname{Cen} A$ by the theorem of $[\mathbf{A}-\mathbf{S}]$.

4.7. Corollary. If $A$ is a maximal commutative $V N R$ subring of a non $V N R$ ring $K$, and if $A^{\prime}$ is generated by idempotents then $A$ is a maximal commutative subring of $K$.

Proof: Since $A \supseteq A^{\prime} \supseteq A$, then $A=A^{\prime}$ is a maximally commutative subring in $K$.

4.8. Corollary. A commutative ring $K$ generated by idempotents is a VNR ring.

Proof: A union of $V N R$ rings is $V N R$, so if we deny the theorem, then $K$ contains a genuine maximal $V N R$ subring $A$. By the corollary, however, $A$ contains $\operatorname{Cen} K=K$.

4.9. Corollary. If $A$ is a maximal commutative VNR subring of a noncommutative $V N R$ ring, and if $\operatorname{Cen} K$ (resp. $A^{\prime}$ ) is generated by idempotents, then $A \supseteq \operatorname{Cen} K$ (resp. $A=A^{\prime}$ is a maximal commutative subring).

\section{Proof: Same.}

We use the concept of a dense submodule $M$ of a right $A$-module $K$, equivalently, $K$ is a rational extension of $M$. The condition is that

$$
\operatorname{Hom}_{A}(T / M, K)=0
$$

for all submodules $T$ of $K$ containing $M$. See [L2] or $[\mathbf{F}],[\mathbf{F} 2]$ for other characterizations, and the background for the following.

$K$ is right torsion free over $A$ provided that no nonzero element of $K$ annihilates a dense right ideal of $A$. When $A$ is right nonsingular, then a right ideal $I$ is dense iff $I$ is an essential right ideal, so torsionfreeness is equivalent to nonsingularity over non-singular rings.

A subring $A$ of $K$ is right dense if $A$ is a dense right $A$-submodule of $K$, and $A$ is left flat if $K$ is a flat left $A$-module. 
4.1' Theorem. If $K$ is a right self-injective ring and right torsionfree over a left flat subring $A$ then $Q=Q_{\max }^{r}(A)$ embeds uniquely as a subring of $K$ containing $A$.

Proof: The proof is the same mutatis mutandi as the proof of Theorem 3.1.

Similarly the following corollaries are proved as the corresponding corollaries above.

$4.3^{\prime} \mathbf{A}$. Corollary. If $K$ is a right self-injective ring torsionfree over a left flat maximal subring $A$, then either $K=Q$, or else $A=Q$.

4.3'B. Corollary. If $A$ is a right dense left flat subring of a right self-injective ring $K$, then $K=Q$.

Corollary $4.3^{\prime} \mathrm{C}$ does not have enough content to restate.

4.3'F. Corollary. If $K$ is a right self-injective ring is right or left torsion free over a flat maximal commutative subring $A$, then either $K=$ $Q$, or $A=Q$.

4.3'G. Corollary. If a right self-injective $K$ has a dense flot maximal commutative subring $A$, then $K=Q$, hence $K$ is commutative and $Q$ is self-injective.

4.10. Proposition. If $K$ is a right self-injective ring, and if a max $V N R$ subring $A$ contains either a faithful left ideal $L$ of $K$ or a dense right ideal $I$ of $K$, then $Q_{\max }^{r}(A)=K$.

Proof: If $0 \neq x \in K$, then $x L \neq 0$, hence $x A \cap A=0$, so $A$ is a right essential subring of $K$, and $Q_{\max }^{r}(A)=K$ by Corollary 3.3B.

Inasmuch as $K$ is a rational extension of $I$, then $K=Q_{\max }^{r}(I)$ and it follows that $K=Q_{\max }^{r}(A)$.

Note. The $\max V N R$ hypothesis is not needed in the second instance.

\section{Right self-injective rings singular over regular subring}

A ring $K$ is left bounded if cvery essential left ideal contains an ideal; $K$ is left strongly bounded (= left $S B$ ) if every nonzero left ideal a nonzero ideal. If $K$ is both lcft and right $S B$, we say that $K$ is $S B$.

A simple Artinian ring $K$ is $S B$, since it has no essential onc-sided ideals $\neq K$, but not left $S B$ since $K$ has no non zero ideals $\neq K$. 
A ring $K$ is left (right) duo if every left (right) ideal is an ideal. A left duo ring is thus trivially left $S B$. A duo ring is both left and right duo, hence $S B$. Any Abelian $V N R K$ is duo, hence $S B$, since, e.g. if $L$ is a left ideal, and $x \in L$, then $K x=K e$ for an idempotent $e \in \operatorname{Cen} K$. Thus, $x \in K e=e K$ so

$$
x K \subseteq K x=K e \subseteq L
$$

proving $L$ is an ideal.

A ring $K$ has bounded index $(=B I) n$ provided that every nilpotent element has index $\leq n$, but at least one has index $n$. Then $K$ is a $B I$ ring, on has $B I$.

A $V N R$ is unit regular $(=U R)$ provided that for every $a \in K$ there is a unit $x \in K$ such that $a x a=a$. Then we say $R$ is a $U V N R$ ring.

A ring $R$ is Dedekind Finite (= directiy finite in [G, p. 49]) if $x y=1$ for $x, y \in R$ iff $y x=1$. Any Abelian $V N R$ ring $K$ is $U R$; any $U V N R$ ring $K$ is $D F$, and any $n \times n$ full matrix ring over an Abelian, or $U R$, or $D F, V N R$ is again $D F$. (See [G, p. 50, prop. 5.2 and 5.3]).

If a ring $K$ has no infinite set of orthogonal idempotents, then $K$ is Dedekind finite by a theorem of Jacobson (see e.g. [F6, p. 85] ff.).

5.1. Remark. If $I$ is an ideal of a ring $K$, then $K$ is $V N R$ iff both $I$ and $K / I$ is $V N R$. Moreover, any (Icft, right) ideal $I_{0}$ of $I$ is a (left, right) ideal of $K$.

Proof: See [G, p.2, Lemma 1.3] for the first statement. The proof shows that if $a \in I$, and if $a x a=a$ for some $x \in K$, then $y=x a x$ is an element of such that aya $=a$. Now let $I_{0}$ be, e.g., a left, idcal of $I$, and $a \in I_{0}$. Then $e=y a$ satisfies $e^{2}=e \in K a$, and then

$$
a e=a y a=a \in K a
$$

so that

$$
K a=K e=(K y) a \subseteq I a \subseteq I_{0},
$$

that is, $I_{0}$ is a left ideal of $K$.

5.2. Proposition. Let $S=\operatorname{Sing} K_{A}$ denote the right singular submodule of a ring $K$ over a right nonsingular subring $A$.

Then:

(1) $S$ is a left ideal of $K$ such that $S A \subseteq S$ and $S \cap A=0$.

(2) If $e=e^{2} \in S$, then $e K \cap A=K e \cap A=0,(1-e) A \approx A$, and $(1-e) A \cap A$ is an essential right ideal of $A$.

(3) If $K$ is injertive in mod-A (e.g., if $K$ is right self-injective, and a flat left $A$-module), then $(1-e) K$ contains an injective hull of $(1-e) A \approx A$ in $\bmod -A$. 
Proof: The singular submodule of a module is fully invariant, so $S$ is a ( $K, A$ )-submodule of $K$ since $K \hookrightarrow$ End $K_{A}$ canonically. Since $A$ is right singular, then $S \cap A=0$, and hence $K e \cap A=0$ and also $e A \cap A=0$. Since $e K \cap A \subseteq e A \cap A$, then $e K \cap A=0$. Thus $(1-e) A \approx A$. Furthermore $(1-e) A \cap A$ is an essential right ideal of $A$ since it is the annihilator in $A$ of $e \in S$. This proves (1) and (2), and (3) follows from injective moduletheory, since, under the hypotheses, $K$ whence $(1-e) K$ is injective over A. (The parenthetic assertion comes from Proposition 1.1).

5.3. Theorem. Let $K$ be a right self-injective $V N R$ ring. The following conditions are equivalent:

(1) $K$ is $B I$.

(2) $K$ is $P A$.

(3) $K / M$ is ortinian for all maximal ideals.

(4) $K$ is isomorphic to a finite product of full matrix rings over abelian regular rings.

In this case $K$ is self-injective.

Proof: See $[\mathbf{G}$, p. 79]. (1) $\Rightarrow(2)$ is a theorem of Utumi, $(1) \Rightarrow(4)$ is a theorem of Kaplansky [K], assuming all $K / M$ have the same index, and Armendariz-Steinberg [A-S] in the general case. (1) $\Rightarrow(3)$ is a remark of Goodearl's (loc. cit.), and the last assertion is a theorem of Ltumi [U2].

5.4. Theorem. Let $L$ be a right self-injective VNR ring.

(1) (Renault) If $K$ is Dedekind finite then: (*) every nonzero ideal contains a central idempotent.

(2) (Utumi [U1]) If $K$ is also left $\left(\aleph_{0}-\right)$ self-injective then $K$ is Dedekind finite, hence $(*)$ holds.

(3) (Utumi) If $K$ is a $P A$, equivalently, $B I$ ring, then $K$ is left selfinjective, hence (*) holds.

Proof: See [G, p. 105, Theorem 9.25 and 9.29] for (1) and (2), and [G, p. 70, Corollary 6.22] for (3). Also see op. cit., pp. 70, 79 and 109 for specific references.

5.5. Theorem. If a right self-injective $V N R$ ring $K$ is right singular over a max $V N R A$, and if $K$ is left strongly bounded, then $A$ is a right self-injective, and the right singular $A$-Submodule $S$ of $K$ is a minimal ideal such that $K / S \approx A$, and

$$
K=S+A \text { and } S \cap A=0 .
$$


Proof: By Proposition 5.2, $S$ is a left ideal of $K$ such that $S \cap A=0$. Let $L$ be a nonzero ideal contained in $S$. Then, $L$ is a regular ideal and by Remark $5.1, L+A$ is a regular ring $\supset A$ so $L+A=K$. Since $S \supset L$, and $S \cap A=0$, then $S=L+(S \cap A)=L$ is a minimal ideal and $K=S+A$. Since $A$ is a direct summand of $K$ in $\bmod -A$, then $A$ is right self-injective by Proposition 1.1 and clearly $K / S \approx A$.

5.6. Theorem. If a right $S I$ Abelian $V N R$ ring $K$ is right singular over a max $V N R$ subring $A$, then $A$ is right $S I$, and isomorphic to a ring direct factor $K_{2}$ of $K$ :

$$
K=K_{1} \times K_{2} \approx K_{1} \times A
$$

where $K_{1}$, the singular right $A$-submodule of $K$ is a shew field.

Proof: $K$ is Abelian, hence $S B$, hence Theorem 5.5 applies $A$ is a right $S I$, and $K_{1} \cap A=0$, where $K_{1}=\operatorname{sing} K_{A}$ is a $(K, A)$-submodule of $K$. Since $K$ is Abelian, hence duo, then every one-sided ideal of $K$ is an ideal. By Theorem 5.5, $K_{1}$ is a minimal ideal of $K$, and by Remark 5.1, every (left, right) ideal $I$ of $K_{1}$ is a (left, right) ideal of $K$, hence $I=K_{1}$. This shows that $K_{1}$ is a skew field.

Moreover, if $e=e^{2} \in K_{1}$ is a nonzero idempotent, then $e$ is central and

$$
K_{1}=e K=K e=e K e
$$

splits off of $K$ as a ring factor:

$$
K=K_{1} \times K_{2}
$$

where $K_{2}=(1-e) K$. Now, since $K / K_{1} \approx A$ by Theorem 5.5 , then $K_{2} \approx A$ as rings, in fact since $K_{1} \cap A=0$, then $e A \cap A=0$, hence

$$
K_{2}=(1-e) K=(1-e) A \approx A .
$$

Finally, by Theorem 5.3 , both $K$ and $A$ are 2-sided $S I$.

Max $K$ denotes the set of maximal ideals of $K$.

5.7. Theorem. Let $A$ be a genuine maximal VNR subring of directly finite right self-injective $V N R$ ring $K$.

(1) If $A$ contains a maximal ideal $M$ of $K$, then $A / M$ is a genuine maximal $V N R$ subring of a simple right self-injective $V N R$ ring $K / M$.

(2) Otherwise

$$
K \underset{\text { subdirect }}{\hookrightarrow} \Pi_{M_{a} \in \max K} K / M_{a} \approx \Pi_{a} A /\left(A \cap M_{a}\right)
$$

i.e., $K$ is isomorphic to a subdirect product of simple homomorphic images of $A$. 
Proof: Theorem 9.32 of [G] implies $K / M$ is right $S I$, so (I) is evident. If (1) fails, then $M_{a}$ is a regular ideal $\nsubseteq A$, hence by Remark $5.1, K=$ $A+M_{a} \in \operatorname{Max} K$. By Corollary 9.26 of $[\mathrm{G}]$, necessarily $\cap_{A} M_{A}=0$, hence $K$ is a subdirect product as stated.

Note. Corollary 9.26 is a consequence of a theorem of F. Maeda. See notes, p. 109 of $[\mathbf{G}]$, where also the origin of Theorem 9.32 is sketched.

\section{Open Questions}

There are many open problems associated with $\max V N R$ subrings of rings, and this paper barely scratches the surface. Here are sample questions.

Does Menal's Theorem hold for the general context of Menal's General Theorem 1.7? Namely, is $A$ or $B$ algebraic over $k$, if $A \otimes_{k} B$ is $S I$ (or VNR)?

Can Theorem 5.6 be extended to a full $n \times n$ matrix $K=R_{n}$ over a right $S I$ Abclian $V N R$ ring $R$, i.e., if $K$ is right-singular over a max $V N R$ subring $A$, is $A$ necessarily $S I$ ? If so, is $A$ isomorphic to a ring direct factor of $K$ ?

What is the Galois Theory for $S I V N R$ subrings of a $V N R$ right $S I$ $\operatorname{ring} K$ ?

By Proposition 1.1, the invariant or Galois subring $A=K^{G}$ is also right $S I$ whenever $K$ is split-flat over $A$. If $|G|=n$ is a unit, then $A$ is $S I$, when ${ }_{A} K$ is flat, e.g. when $A$ is $V N R$ (Corollary 1.2A). This and Corollary $1.2 \mathrm{~B}$ generalizes the theorem of $\mathrm{A}$. Page $[\mathrm{P}]$.

\section{Pere Menal i Brufal: A Memento}

It would be impossible to fully express in this brief space the debts of friendship, both personal and mathematical, that I owe to the late Pere Menal.

It all began in 1981 when I wrote to Pere about his work [M] that I discussed in the Abstract on algebraic regular rings in connection with tensor products. He expressed amazement and delight that it was connected with the Hilbert Nullstellensatz.

After some years of correspondence, we began contemplating an algebra semester under the auspices of Manuel Castellet's CRM, and we went on to organize one in Spring 1986; another much larger conference followed in Fall 1989.

Much creative mathematics flowed out of these conferences, particularly the theorem of Pere Menal and Peter Vámos [M-V] that realized 
a three decades-old dream of ring theory: an embedding of any ring in an $F P$-injectve ring ${ }^{1}$.

In the 1989 semester, Pere and I collaborated on a problem that eluded us individually for many years, and we finally found that what we were looking for: "A counter-example to a conjecture of Johns" which just appeared in the Proceedings of the American Mathematical Society (1992).

These are just two of the myriad collaborations that Pere Menal had with others: Jaume Moncasi, Pere Ara, Claudi Busqué, Ferran Cedó, Dolors Herbera, Rosa Camps (his talented students and colleagues) and Brian Hartley, Warren Dicks, Kenneth Goodearl, the aforementioned Peter Vámos, Boris Vaserstein (to mention but a few of his intense interactions with others).

In particular, Pere's paper with Dolors Herbera [H-M], Ferran Cedó paper $[\mathrm{C}]$, Dolors Herbera's paper with Poobhalan Pillay [H-P], and her Doctoral Thesis at L.A.B., each greatly advanced our knowledge on subjects taken up in seminars during these conferences.

I am grateful that I happened to write to Pere back in 1981; otherwise, I might never have met this noble and gentle genius of Catalunya, who became an inspiration to so many.

Wherever Pere is now, I like to think that he is continuing his work that he left here on earth, and collaborating with the Great One.

\section{References}

[Az] G. Azumaya, On maximally central algebras, Nagoya Math. $J$. 2 (1951), 119-150.

[A-O] M. AUSLANDER AND O. GolDMAN, The Brancr group of a commutative ring, Trans. A.M.S. 97 (1960), 367-409.

[A-S] E. ARMENDARIZ AND S. STEINBERG, Regular self-injective rings with PI, Trans. Amer, Math. Soc. 190 (1974), 417-425.

[B] H. BAss, "Lectures on topics in algebraic K-theory," Tata Inst. for Advanced Study, Colaba, Bombay, 1967.

[Bu] C. BusquÉ, Centers and two-sided ideals in right self-injective regular rings, Journal of Algebra, to appear.

[C-E] H. Cartan and S. Eilenberg, "Homological Algebra," Princeton, 1956.

\footnotetext{
${ }^{1}$ In her Rutgers University ph.D. thesis (1964), Barbara Osofsky had shown that in general the injective hull of a ring $R$ could not be made into a ring containing $R$ as a subring. It is also known that $R$ can not always be embedded in a self-injective ring.
} 
[C] F. CEDó, Zip rings and Mal'cev domains, Comm. Alg. 19 (1991), 1983-1991.

[F] C. FAITH, "Injective Modules and Quotient rings," Lecture Notes in Math. 49, Springer-Verlag, 1967.

[F2] C. FAITH, "Injective modules and injective quotient rings," Lecture Notes in Pure and Applied Math. 72, Marcel Dekker, 1982.

[F3] C. FAITH, Subrings of self-injective and FPF rings, in "Advances in Non-Commutative ring theory," Lecture Notes in Math. 951, 1982, pp. 12-20.

[F4] C. FAITH, The maximal regular ideal of self-injective and continuous rings splits off, Arch. der Math. 44 (1985), 511-521.

[F5] C. FarTH, "Algebra I: Rings, Modules and Categories," Springer Vcrlag, Berlin, Heidelberg, New York, 1973. Corrected Reprint 1981.

[F6] C. FaIth, "Algebra II: Ring Theory," Springer Verlag, 1976.

[F-M] C. Faith and Pere Menal, A counter-example to a conjecture of Johns, Proc. Amer. Math. Soc. 116 (1992), 21-26.

[F-P] C. FAITH AND S. PAGE, "FPF Ring Theory: Faithful modules and generators of mod- $R, "$ Lecture Notes of the London Math. Soc. 88, Cambridge University Press, Cambridge, London, New York, Melbourne, Sydney, 1984.

[G] K. R. GoodearL, "Von Neumann regular rings," Pitman, 1979.

[H] DOLORS HFRBfRA, On rings whose finitely generated faithful modules are generators, $\mathrm{Ph}$.D. Thesis (in catalan), Universitat Autònoma de Barcelona, 08193 Bellaterra, Barcelona (1992).

[H-M] Dolors HfrRfia AND Pfre Menal, On rings whose finitely generated faithful modules are generators, J. of Algebra 122 (1989), $425-438$.

[H-P] Dolors Herbera AND P. PILlay, Injective classical rings of quotients of the polynomial rings are quasi-Frobenius, $J$. Pure and Applied Algebra, to appear.

$[\mathrm{J}]$ Nathan JaCOBSON, "The Structure of rings," American Math. Society, Colloquium Publication 37, Providence, R.I., 02940, 1956 (rev. ed. 1964).

[K] I. KaPLANSKY, Topological representation of algebras, Trons. Amer, Moth. Soc. 68 (1950), 62-75.

[L] J. LAMBEK, A module is flat iff its character module is injective, Canad. Math. Bull 7 (1964), 237-243.

[L2] J. LAMBEK, "Lectures on rings and modules," Chelsea, New York. 
[M] P. MENAL, On tensor products of algebras being Von Neumann regular or self-injective, Comm. Alg. 9(7) (1981), 691-697.

[M-V] P. Menal and P. VÁmos, Pure ring extensions and self- $F P$ injective rings, Math. Proc. Cambridge Philos. Soc. 105 (1989), 447-458.

[P] A. PAGE, "Actions de groupes," Sem. P. Dubreil (1977-78), Lecture Notes in Math. 740, Springer Verlag, 1979.

[R] R. L. Rao, Azumaya, Semisimple and ideal algebras, Bull. Amer. Math. Soc. 78 (1972).

[Re] G. REnAult, Anneaux reguliers auto-injectifs a droite, Bull. Soc. Math. France 101 (1973), 237-254.

[Re2] G. Renault, Anneaux reguliers auto-injectifs a droite, $J$. of Algebra 36 (1975), 77-84.

[Re3] G. RENAULT, "Actions des groupes et anneaux reguliers injectifs," Proc. Conf. Waterloo (1978), Lecture Notes in Math. 734, Springer-Verlag, 1979.

[S] G. Szero, A characterization of Azumaya algebras, J. Pure and Appl. Alg. 9 (1976/77), 369-371.

[U1] Y. UTUMY, On continuous rings and self-injective rings, Trans. Amer. Math. Soc. 118 (1965), 158-173.

[U2] Y. UTUMI, On self-injective rings, J. Algebra 6 (1967), 56-64.

[U3] Y. UTUMI, On rings of which any one-sided quotent rings are two-sided, Proc. Amer. Math. Soc. 14 (1963), 141-147.

[U4] Y. UTUMI, On continuous regular rings and semisimple selfinjective rings, Canad. J. Moth. 12 (1960), 597-605.

[U5] Y. Utumi, On quotient rings, Osaka Math. J. 8 (1956), 1-18.

Hill Center for Mathematical Sciences

Department of Mathematics

Rutgers, The State University

New Brunswick, NJ 08903

L.S.A.

Rebut el 22 de Gener de 1992 